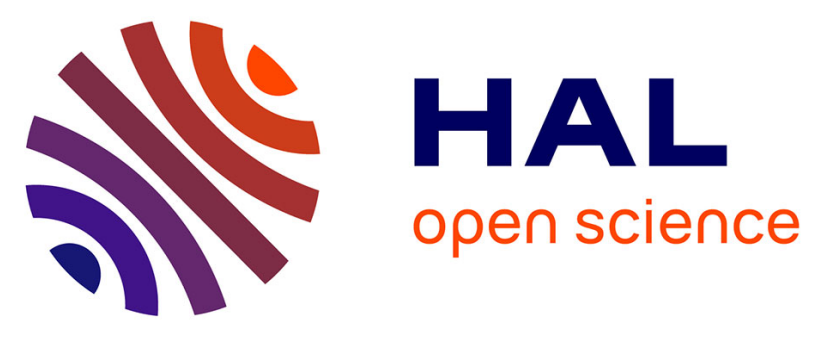

\title{
Cutaneous Kikuchi disease-like inflammatory pattern without lymph node involvement is associated with systemic disease and severe features in lupus erythematous: A case-control study
}

Sophie Lalevée, Philippe Moguelet, Charlotte Hurabielle, Patricia Senet, Micheline Pha, Sébastien Rivière, Annick Barbaud, Zahir Amoura, François Chasset, Camille Francès

\section{- To cite this version:}

Sophie Lalevée, Philippe Moguelet, Charlotte Hurabielle, Patricia Senet, Micheline Pha, et al.. Cutaneous Kikuchi disease-like inflammatory pattern without lymph node involvement is associated with systemic disease and severe features in lupus erythematous: A case-control study. Lupus, 2021, 30 (3), pp.473-477. 10.1177/0961203320978519 . hal-03184139

\section{HAL Id: hal-03184139 \\ https://hal.sorbonne-universite.fr/hal-03184139}

Submitted on 29 Mar 2021

HAL is a multi-disciplinary open access archive for the deposit and dissemination of scientific research documents, whether they are published or not. The documents may come from teaching and research institutions in France or abroad, or from public or private research centers.
L'archive ouverte pluridisciplinaire HAL, est destinée au dépôt et à la diffusion de documents scientifiques de niveau recherche, publiés ou non, émanant des établissements d'enseignement et de recherche français ou étrangers, des laboratoires publics ou privés. 


\section{Cutaneous kikuchi disease-like inflammatory pattern without lymph node involvement is associated with systemic disease and severe features in lupus erythematous: a case-control study.}

Running title: Cutaneous kikuchi disease-like inflammatory pattern in lupus

S. Lalevée ${ }^{*}, \mathrm{MD}, \mathrm{P}$. Moguelet $^{1 *}, \mathrm{MD}, \mathrm{C}$. Hurabielle$^{2}, \mathrm{MD}, \mathrm{PhD}, \mathrm{P} . \mathrm{Senet}^{3}, \mathrm{MD}, \mathrm{M} . \mathrm{Pha}^{4}, \mathrm{MD}$, S. Rivière 5 , MD, A. Barbaud ${ }^{3,6}, \mathrm{MD}, \mathrm{PhD}$, Z. Amoura ${ }^{4}, \mathrm{MD}, \mathrm{PhD}, \mathrm{C}$. Francès ${ }^{3}, \mathrm{MD}, \mathrm{PhD}, \mathrm{F}$. Chasset $^{3}, \mathrm{MD}, \mathrm{PhD} .{ }^{*}$ contributed equally to the manuscript as co-first author.

${ }^{1}$ Service d'anatomopathologie, Hôpital Tenon, Assistance Publique-Hôpitaux de Paris,75020 Paris, France ; ${ }^{2}$ UCSF Internal Medicine, 505 Parnassus Avenue, San Francisco, CA 94143, USA ; ${ }^{3}$ Service de Dermatologie et Allergologie, Hôpital Tenon, Sorbonne Université, Faculté de Médecine, AP-HP, 75020 Paris, France $;{ }^{4}$ Service de Médecine Interne 2, Centre National de Référence du lupus systémique, Hôpital Pitié-Salpêtrière, Assistance Publique-Hôpitaux de Paris, 47-83, boulevard de l'Hôpital, 75651, Paris Cedex 13, France ; ${ }^{5}$ Sorbonne Université, Faculté de Médecine, AP-HP, Service de médecine interne, Hôpital Saint-Antoine F-75012, Paris, France ; ${ }^{6}$ INSERM, Institut Pierre Louis d'Epidémiologie et de Santé Publique, Médecine Sorbonne Université 
Corresponding author: François Chasset

Service de Dermatologie et d'Allergologie, Hôpital Tenon,

4 rue de la Chine 75970, Paris Cedex 20, France

Tel +33 156017547; Fax +33 156016702 ; Email: francois.chasset@aphp.fr

Key-words: Kikuchi-Fujimoto disease, kikuchi disease-like inflammatory pattern, cutaneous lupus erythematosus, systemic lupus erythematosus

No author has a conflict of interest regarding this manuscript.

Words count: 1454 Figure : 1 Table count: 2 References count: 10 


\section{INTRODUCTION}

Kikuchi-Fujimoto disease (KFD) is a self-limited histiocytic necrotizing lymphadenitis that typically affects the cervical lymph nodes and sometimes the skin. An immune response of T cells and histiocytes to an infectious agent is considered to be the most probable cause but association with autoimmune diseases particularly with systemic lupus erythematosus (SLE) has been reported in 12 to $36 \%$ of cases ${ }^{1,2}$. KFD associated with SLE differed from isolated KFD with a higher frequency of severe forms and extranodal manifestations including skin involvement ${ }^{3}$. Importantly, KFD may occur before the diagnosis of SLE in up to $30 \%$ cases ${ }^{3}$ and some authors suggested that skin involvement may be a risk factor of developing SLE among KFD patients ${ }^{1}$. Predominant histologic findings of KFD in the skin are variable degrees of interface dermatitis and dermal lymphohistiocytic infiltrate with non-neutrophilic karyorrhexis ${ }^{4}$. Recently, Thai and al. described "Kikuchi disease-like inflammatory pattern" (KLIP) in cutaneous lesions showing histological features similar to KFD without lymph node involvement ${ }^{5}$. In their cohort $67 \%$ of patients had either isolated cutaneous lupus (CLE) or SLE. In order to better characterize this entity, we aimed to describe clinical, immunological, pathological features, and the response to treatment of lupus patients with KLIP observed in the skin biopsies. We particularly intended to assess whether KLIP was associated with SLE, severe systemic features and the risk of progression from isolated CLE to SLE. 


\section{MATERIAL AND METHODS}

To describe KLIP-associated features in lupus patients, we performed a retrospective case-control study including patients with or without KLIP on skin biopsies. Patients with lymphadenopathy were excluded. In order to assess whether KLIP was associated with severe skin involvement, SLE, SLE severe features and the risk of progression from isolated CLE to SLE, age- and sexmatched control lupus patients (3/1) without KLIP were included, identified from our informatic pathological database blindly from clinical data. CLE subtypes were classified according to clinical presentation, epidermal abnormalities, and dermal lymphocytic pattern ${ }^{6,7}$ (subacute cutaneous lupus erythematosus (SCLE), discoid or tumidus lupus). SLE was diagnosed according to Systemic Lupus International Collaborating Clinics (SLICC) criteria ${ }^{4}$. Severe SLE was defined by the presence of at least one severe systemic feature among lupus nephritis, serositis or haematological lupus (immune thrombocytopenia purpura or autoimmune hemolytic anemia). KLIP was defined as a dermal infiltrate or foci within dermal infiltrates, composed of mononuclear cells $(\mathrm{CD} 163+$ macrophages including some MPO+ cells, CD123+ plasmacytoid dendritic cells and CD8+ lymphocytes including some cytotoxic granzyme B+ cells) and nuclear debris without neutrophils ${ }^{5}$ (Figure 1). For each case, KLIP was determined as extensive or localized. Data are presented as median (range) or counts (percentage). Statistical analyses were performed using Fisher's exact test and odds ratio (OR) with 95\% confidence intervals were estimated. A 2-tailed $\mathrm{p}$ value $<0.05$ was considered statistically significant. Analyses were performed with JMP software (version 14 [SAS Institute Inc, Cary, NC]). 


\section{RESULTS}

\section{Patient and control characteristics}

Thirteen cases with KLIP on their skin biopsies (all women) were included (median age 37, range 20-52 years). Nine of these 13 women were from non-caucasian ethnic backgrounds. Moreover, thirty-nine age- and sex-matched control lupus patients without KLIP were included.

At the time of KLIP diagnosis, 4/13 patients (31\%) had isolated CLE and 9/13 had (69\%) SLE including 6 (46\%) with severe haematological, lung, cardiac or renal disease (Table 1). KLIP features were observed in biopsies of different clinical presentations, discoid, tumidus, subacute or nonspecific lesions (Table 1) and was not associated to a specific clinical presentation.

In the control group $31 / 39$ patients $(79 \%)$ had isolated CLE (discoid $(n=20)$, subacute $(n=10)$, tumidus $(\mathrm{n}=8)$ and panniculitis $(\mathrm{n}=1)), 8 / 39(21 \%)$ had SLE including 3 with severe SLE. Biologically all patients showed anti-nuclear-antibodies (ANA) except one CLE for whom the results were not available.

\section{Pathological features of KLIP}

Considering pathological findings, epidermal appearance and dermal lymphocytic pattern allowed the histological classification as lupus lesions. KLIP was always associated with other features of lupus: interface dermatitis $(n=13)$, mucin deposition $(n=10)$, clusters of CD123+ dendritic cells $(n=13)$. In immunohistochemistry, myeloperoxidase (MPO) expression was the most useful criteria for the diagnosis of KLIP, abundant in extensive forms, focal in localized forms. The KLIP pattern was extensive $(n=9)$ or localized in the superficial dermis $(n=4)$. 
Histological findings were not significantly different in isolated CLE compared with SLE patients (data not shown).

\section{Association of KLIP with SLE, severe SLE and risk of progression from CLE to SLE}

As shown in the Table 2, KLIP patients more frequently had SLE 9/13 (69\%) compared to our control group 8/39 (21\%) (OR 8,72; CI95\% [2,13 -35,75]; $\mathrm{p}=0.002)$ and more frequently severe SLE with 5/13 patients (38\%) compared to $3 / 39$ (8\%) (OR 7,5; CI95\% [1,48-38,03]; $\mathrm{p}=0.017)$. Moreover, two out of four CLE exhibiting KLIP lesions (50\%) developed severe SLE with cardiac or renal involvement after 12 and 24 months, respectively. In contrast, in the control group no CLE developed SLE (2/4 vs 0/31; p=0.01) after a median follow-up of 84 months, range 12-360.

\section{Therapeutic management of KLIP cutaneous lesions}

Interestingly, KLIP flares occurred while on systemic corticosteroids $(n=6)$ or immunosuppressive agents $(n=4)$ including belimumab $(n=1)$, mycophenolate mofetil $(n=1)$ or methotrexate ( $\mathrm{n}=2)$ (Table 1). A trigger for the flare was identified in $8 / 13$ patients $(61 \%)$ (Table 1). Only one patient had spontaneous resolution of cutaneous lesions without treatment. Two patients responded well to topical steroids alone. Two patients with CLE were efficiently treated with topical steroids associated with antimalarials. Increasing corticosteroids dose was effective in 2 SLE patients. Treatment with thalidomide $100 \mathrm{mg}$ /day allowed rapid clearance of cutaneous lesions in 6/6 KLIP patients. The need to use thalidomide tended to be more frequent in KLIP patients compared with patients without KLIP (OR 3;2; CI95\% [0.87-11.75]; $\mathrm{p}=0.07)$ (Table 2). 


\section{DISCUSSION}

In this study we aimed to portray KLIP features (i.e similar cutaneous pathological features than those of KFD but without lymph node involvement) among CLE and SLE patients.

In our study KLIP was observed exclusively in female patients, with a median age of 37 years and with a predominance of non-caucasian ethnic background. This suggest that KLIP arises in a population which does not differ from usual lupus population regarding age, gender or ethnicity 8.

Importantly, KLIP was observed in clinical lesions suggestive of classical CLE subtypes including discoid, subacute or tumidus lupus but also in non-specific maculo-papular eruptions. These findings are in accordance with the study of Thai et al. ${ }^{5}$ showing that the clinical features of KLIP are very heterogeneous.

In the study of Thai et al, among 16 lupus patients, $8(50 \%)$ had extracutaneous symptoms ${ }^{5}$. In our series, 9/13 had (69\%) SLE and interestingly, 6 (46\%) had severe haematological, lung, cardiac or renal involvement. In order to assess whether KLIP was associated with SLE and severe systemic features with selected 3/1 age- and sex-matched control lupus patients without KLIP identified from our informatic pathological database blindly from clinical data. As shown in Table 2, KLIP patients had significantly more frequently SLE and severe SLE features compared with lupus patients without KLIP.

Some studies suggest that classical KFD may precede SLE ${ }^{1,3}$. Indeed, in a systematic review including 158 adults with proven KD associated with SLE, the diagnosis of SLE was done after 
$\mathrm{KD}$ in $31 \%$ of cases ${ }^{3}$. Moreover, in a prospective study, the risk of developing moderate to severe SLE in isolated CLE patients was only 5/77 (6\%) ${ }^{9}$. Importantly, two of our four patients $(50 \%)$ with CLE exhibiting KLIP lesions secondarily developed SLE with severe cardiac or renal involvement compared with $0 / 39$ in the control group $(p=0.01)$. As Thai et al. suggested KLIP could be a clue for the diagnosis of lupus ${ }^{5}$, we suggest that it may be a clue for progression from CLE to SLE although our limited sample size prevents definite conclusions.

Although KFD classically require no specific treatment ${ }^{2}$ most cutaneous flare of KLIP occurred in lupus patients while on systemic corticosteroids or immunosuppressive treatment. This suggest that KLIP may be a clue for refractory cutaneous involvement in lupus patients. This finding is supported by the fact that KLIP patients tended to be more treated with thalidomide than control lupus patients $(\mathrm{p}=0.07)$. Indeed, thalidomide has shown excellent results for severe CLE with overall response rate of $90 \%$ but its use is limited by potentially severe adverse events and therefore its prescription is limited to severe CLE patients ${ }^{10}$. Interestingly, as for all other CLE subtypes ${ }^{10}$, thalidomide was effective in KLIP lesions and may therefore be a good therapeutic option.

Overall, this study suggests that KLIP features in lupus skin lesions are associated with SLE and severe systemic features. Despite a limited number of isolated CLE patients with KLIP features in the skin, they may warrant closer follow-up on patients with a higher risk of developing SLE. 


\section{References:}

1. Dumas G, Prendki V, Haroche J, Amoura Z, Cacoub P, Galicier L, et al. Kikuchi-Fujimoto disease: retrospective study of 91 cases and review of the literature. Medicine (Baltimore). 2014 Nov;93(24):372-82.

2. Moinet F, Molinié V, Béraud G, Polomat K, Cordel N, Sainte-Marie D, et al. Epidemiology and Characteristics of Kikuchi-Fujimoto Disease in the African-Descent Population of Martinique, French West Indies. Arthritis Care Res. 2016;68(12):1883-7.

3. Sopeña B, Rivera A, Chamorro A, Freire M, Alende V, Seco E, et al. Clinical association between Kikuchi's disease and systemic lupus erythematosus: A systematic literature review. Semin Arthritis Rheum. 2017;47(1):46-52.

4. Kim JH, Kim YB, In SI, Kim YC, Han JH. The cutaneous lesions of Kikuchi's disease: a comprehensive analysis of 16 cases based on the clinicopathologic, immunohistochemical, and immunofluorescence studies with an emphasis on the differential diagnosis. Hum Pathol. 2010 Sep;41(9):1245-54.

5. Thai L-H, Ingen-Housz-Oro S, Godeau B, Rethers L, Wolkenstein P, Limal N, et al. Kikuchi Disease-Like Inflammatory Pattern in Cutaneous Inflammatory Infiltrates Without Lymph Node Involvement: A New Clue for the Diagnosis of Lupus? Medicine (Baltimore). 2015 Nov;94(46):e2065.

6. Kuhn A, Landmann A. The classification and diagnosis of cutaneous lupus erythematosus. J Autoimmun. 2014 Mar;48-49:14-9.

7. Baltaci M, Fritsch P. Histologic features of cutaneous lupus erythematosus. Autoimmun Rev. 2009 May;8(6):467-73.

8. Pons-Estel GJ, Ugarte-Gil MF, Alarcón GS. Epidemiology of systemic lupus erythematosus. Expert Rev Clin Immunol. 2017;13(8):799-814.

9. Wieczorek IT, Propert KJ, Okawa J, Werth VP. Systemic symptoms in the progression of cutaneous to systemic lupus erythematosus. JAMA Dermatol. 2014 Mar;150(3):291-6.

10. Chasset F, Tounsi T, Cesbron E, Barbaud A, Francès C, Arnaud L. Efficacy and tolerance profile of thalidomide in cutaneous lupus erythematosus: A systematic review and metaanalysis. J Am Acad Dermatol. 2018;78(2):342-350.e4. 


\section{Table 1: Characteristics of the 13 KLIP patients}

* ACLE : acute cutaneous lupus erythematosus, AIHA: autoimmune hemolytic anemia, ANA: Antinuclear autoantibodies, anti-dsDNA: anti- double stranded DNA antibodies, aPL: antiphospholipid antibodies, CQ: chloroquine, DLE : discoid lupus erythematosus, GC: glucocorticoids, HCQ: hydroxychloroquine, LN: Lupus Nephritis, MMF: Mycophenolate mofetil, PV: perivascular, RNP : ribonucleoprotein, SCLE : subacute cutaneous lupus erythematosus, SLE : systemic lupus erythematosus, SSA / SSB : Sjögren's-syndrome-related antigen A / B, TCS: Topical corticosteroids.

Table 2: Statistical analysis comparing KLIP cohort $(n=13)$ to $3 / 1$ age- and sex-matched control lupus patients without KLIP (n=39)

\begin{tabular}{l|cccc}
\multicolumn{1}{l}{ KLIP+ } & KLIP- & OR ; 95\% CI & P-VALUE \\
\hline SYSTEMIC LUPUS & $9 / 13(69 \%)$ & $8 / 39(21 \%)$ & $8.72 ;[2.13-35.75]$ & $\mathbf{0 . 0 0 2}$ \\
& & & & \\
$\begin{array}{l}\text { SEVERE SYSTEMIC } \\
\text { LUPUS }\end{array}$ & $5 / 13(38 \%)$ & $3 / 39(7 \%)$ & $7.5 ;[1.48-38.03]$ & $\mathbf{0 . 0 1 7}$ \\
$\begin{array}{l}\text { EVOLUTION FROM } \\
\text { ISOLATED CLE TO }\end{array}$ & $2 / 4(50 \%)$ & $0 / 31(0 \%)$ & $\infty ;[1.69-\infty]$ & $\mathbf{0 . 0 1}$ \\
$\begin{array}{l}\text { SLE } \\
\text { NEED FOR } \\
\text { THALIDOMIDE }\end{array}$ & $8 / 13(61 \%)$ & $13 / 39(33 \%)$ & $3.2 ;[0.87-11.75]$ & 0.07 \\
\end{tabular}

KLIP: Kikuchi disease-like inflammatory pattern; OR: odds ratio; CI: confidence intervals 


\section{Figure 1: Histological presentation of KLIP in lupus patients}

a, b, $\mathbf{c}, \mathbf{d}$ : extensive KLIP pattern $\left(1^{\text {st }}\right.$ patient $\left.35 / F\right)$ as seen in $n=9$ patients; $\mathbf{e}, \mathbf{f}, \mathbf{g}, \mathbf{h}:$ localized KLIP pattern $\left(4^{\text {th }}\right.$ patient $\left.40 / F\right)$ as seen in $n=4$ patients. KLIP pattern: dermal infiltrate or foci within dermal infiltrates, composed of mononuclear cells and nuclear debris without neutrophils.

a, b, c, e, f, g: hematoxylin- eosin-saffron (HES)-stained; a, e: original magnification x25: superficial and deep dermal infiltrate; b, f : original magnification x200: interface dermatitis, perivascular and interstitial infiltrate; $\mathbf{c}, \mathbf{g}$ : original magnification x1000: plasmacytoid dendritic cells and nuclear debris without neutrophils; d, h: Immunoperoxydase stain for MPO; original magnification x100: extensive (d) or localized (h) expression. 